\title{
A Influência das Características dos Prestadores e dos Utentes no Consumo de Recursos em Unidades de Cuidados Continuados
}

\author{
Rui Santana ${ }^{a, b}$ Ana Patrícia Marques ${ }^{a}$ Sílvia Lopes ${ }^{a, b} \quad$ Paulo Boto ${ }^{a, b}$ \\ José Luis Telles ${ }^{c}$ Sónia Félix ${ }^{d, e}$ Ricardo Mestre ${ }^{f}$ Rosa Matos ${ }^{g}$ Bruno Moita ${ }^{h}$ \\ ${ }^{a}$ Centro de Investigação em Saúde Pública da Universidade NOVA de Lisboa, Lisbon, Portugal; bepartamento de \\ Políticas e Gestão dos Sistemas de Saúde, Escola Nacional de Saúde Pública, Universidade NOVA de Lisboa, Lisbon,

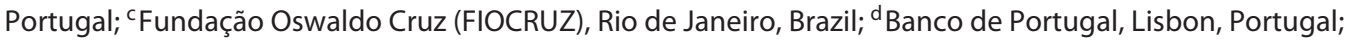 \\ eNOVA School of Business and Economics, Universidade NOVA de Lisboa, Lisbon, Portugal; ${ }^{\dagger}$ Administração Central \\ do Sistema de Saúde, Ministério da Saúde, Lisbon, Portugal; ${ }^{9}$ Administração Regional de Saúde de Lisboa e Vale

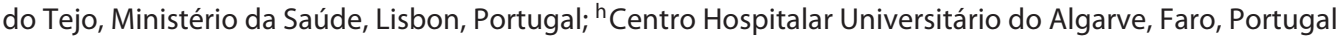

\section{Palavras Chave \\ Organização e gestão dos serviços de saúde · Cuidados continuados · Custos em organizações de saúde}

\section{Resumen}

Introdução: O envelhecimento populacional, o aumento da prevalência de doenças crónicas e de multipatologia, são fenómenos que encontraram novas respostas com a criação da Rede Nacional de Cuidados Continuados Integrados (RNCCI) em Portugal, a partir de 2006. É esperado que esta estrutura adicional de oferta de cuidados permita contribuir para a criação de valor aos seus utentes. Objetivo: O presente estudo teve como objetivos estimar o consumo de recursos medido através da duração de internamento em unidades de internamento em cuidados continuados (UICC) em Portugal e analisar a associação com as características dos utentes e dos prestadores de cuidados. Método: Foi realizado um estudo transversal e retrospetivo, que utilizou informação da atividade das unidades da RNCCI entre 2010 e 2012. Recorreu-se a modelos de regressão lineares múltiplos, utilizando a duração de internamento como variável dependente e, como preditores, variáveis representativas das características individuais dos utentes e dos prestadores. Resultados: Para os 30.090 episódios incluídos, a duração média de internamento foi de 34,2 dias nas unidades de convalescença, 84,1 dias nas unidades de média duração e reabilitação e 106 dias nas unidades de longa duração e manutenção. A dispersão da duração de internamento foi elevada em todas as tipologias e regiões. Isoladamente, as variáveis associadas às características dos utentes apresentaram capacidade preditiva muito reduzida. A inclusão das variáveis associadas à organização da oferta de cuidados aumentou a capacidade do modelo explicar a variabilidade do tempo de internamento dos utentes. Conclusão: Os resultados do modelo de regressão linear múltipla sugerem que são as características associadas à oferta de cuidados que apresentam maior relevância para explicar a variabilidade da duração de internamento em cuidados continuados. Sugere-se que futuros desenvolvimentos incluam melhorias nas práticas de registo e a implementação de um sistema de classificação de utentes específico, internacionalmente validado para a estratificação do risco em cuidados continuados.

(c) 2017 The Author(s). Published by S. Karger AG, Basel on behalf of Escola Nacional de Saúde Pública

\section{KARGER}

E-Mail karger@karger.com www.karger.com/pjp

Karger Open access (c) 2017 The Author(s). Published by S. Karger AG, Basel on behalf of Escola Nacional de Saúde Pública

This article is licensed under the Creative Commons AttributionNonCommercial-NoDerivatives 4.0 International License (CC BYNC-ND) (http://www.karger.com/Services/OpenAccessLicense) Usage and distribution for commercial purposes as well as any distribution of modified material requires written permission.
Prof. Rui Santana, PhD

Escola Nacional de Saúde Pública, Universidade NOVA de Lisboa Avenida Padre Cruz

PT-1600-560 Lisbon (Portugal)

E-Mail ruisantana@ensp.unl.pt 
The Influence of Providers' and Patients' Characteristics on Resource Use in Long-Term Care Inpatient Units

\section{Keywords}

Health services organization and management .

Continuity of care $\cdot$ Costs in health organizations

\section{Abstract}

Introduction: For the aging population, as well as for the growing prevalence of chronic diseases and multipathology, new answers have been found with the creation of the Portuguese National Long Term Care Network (PNLTCN) in 2006. It is expected that this additional level of care provision can create value for patients. Objective: This study aimed to estimate resource use in long-term care inpatient units in Portugal and to analyze its association with providers' and patients' characteristics. Methods: We conducted a cross-sectional retrospective study using information from the PNLTCN units between 2010 and 2012. Multiple linear regression analyses were performed, including the logarithm of length of stay as dependent variable and variables on patients' and providers' characteristics as predictors. Results: For the 30,090 admissions included, the average length of stay was 34.2 days for Convalescent Care Units, 84.1 days for Medium Stay and Rehabilitation Units, and 106 days for Long Stay and Maintenance Units. Significant dispersion was found for all regions and type of providers. Patients' characteristics alone showed low predictive capacity. The inclusion of providers' characteristics increased the model's predictive ability to explain the length of stay. Conclusions: Multiple regression model results suggest that resource utilization in long-term care is mainly influenced by the characteristics of the providers. Future development should include improvements on data collection procedures and the implementation of a risk-adjusted patient classification system internationally validated for long term care.

(c) 2017 The Author(s). Published by S. Karger AG, Basel on behalf of Escola Nacional de Saúde Pública

\section{Introdução}

A necessidade crescente de resposta dos sistemas de saúde a utentes que se encontram na quarta idade, com multipatologia crónica e dependência, é uma realidade presente em todo o mundo, nomeadamente em países

Consumo de Recursos em Unidades de Cuidados Continuados como a Alemanha, Canadá, Estados Unidos da América (EUA), Espanha e Reino Unido [1-6]. Esta tendência desperta o interesse e a atenção por parte dos vários atores e intervenientes do sistema, particularmente no que diz respeito, a novas formas de organização da prestação de cuidados, no seu enquadramento legal, bem como nos mecanismos para melhorar a efetividade e a distribuição equitativa dos recursos e benefícios [7].

Em Portugal, a resposta a este tipo de necessidades materializou-se na criação da Rede Nacional de Cuidados Continuados Integrados (RNCCI). A RNCCI constitui um "conjunto de intervenções sequenciais de saúde e/ou de apoio social, decorrente de avaliação conjunta, centrado na recuperação global" [8] da pessoa em situação de dependência. Esta recuperação pode ser entendida como o "processo terapêutico e de apoio social, ativo e contínuo, que visa promover a autonomia melhorando a funcionalidade da pessoa em situação de dependência através da sua reabilitação, readaptação e reinserção familiar e social" [8]. A prestação de cuidados integrados na RNCCI é desenvolvida por quatro tipos de serviços distintos: Unidades de Internamento, Unidades de Ambulatório, Equipas Hospitalares e Equipas Domiciliárias. No que diz respeito às Unidades de Internamento estas subdividemse em Unidades de Convalescença, Unidades de Média Duração e Reabilitação, Unidades de Longa Duração e Manutenção e Unidades de Cuidados Paliativos [8].

O conceito internacional que se encontra mais próximo do que se entende no nosso contexto por "Cuidados Continuados Integrados" é o de Long-Term Care (LTC). Contudo, a definição de LTC não é unânime no seu âmbito e delimitação. Uma dessas definições, a da Organização para a Cooperação e Desenvolvimento Económico (OCDE), considera LTC como "a range of services for persons who are dependent on help with activities of daily living over an extend period of time" [9]. Por facilidade de abordagem, assumir-se-á o termo Cuidados Continuados (CC) como proxy de LTC independentemente das particularidades existentes em cada país.

Desde a sua criação, em 2006, a RNCCI apresentou um crescimento significativo até ao presente. Este facto pode ser evidenciado pelo número de camas existentes - de 646 em 2006 para 7.160 em 2014 - e pelo seu orçamento global que provém conjuntamente do Ministério da Saúde e Ministério do Trabalho, Solidariedade e Segurança Social - de 3,2 milhões orçamentados em 2006 aumentou para 152,7 milhões no ano de 2014 [10]. Este montante representa cerca de $2 \%$ do orçamento total do Serviço Nacional de Saúde (SNS) e 0,1\% do Produto Interno Bruto (PIB) português. Apesar do crescimento, Portugal não se 
encontra nos padrões de afetação de verbas observados em países como a Alemanha, Reino Unido ou Austrália, onde os valores se aproximam de $1 \%$ do PIB $[5,11,12]$.

Desta forma, a representatividade do fenómeno e o seu previsível crescimento obriga a intervenções cada vez mais custo-efetivas por parte dos diferentes agentes envolvidos nas políticas e gestão dos CC. No entanto, não existem mecanismos uniformes e sistemáticos de recolha, análise e tratamento da informação de custos em CC no nosso país, particularmente no que respeita à sua componente analítica. Noutros contextos, a partilha deste tipo de informação é condição essencial para as organizações poderem desenvolver os seus serviços [13]. Face a esta indisponibilidade de informação de custos, a definição dos preços a aplicar na RNCCI refletiu a expectativa de consumo de recursos estabelecida por um conjunto de peritos em função das características estruturais dos prestadores de cuidados.

Decorridos cerca de 10 anos da implementação da RNCCI, torna-se premente a avaliação das características específicas e diferenciadoras do perfil dos utentes e a sua influência no consumo de recursos. Entre as características consensualmente mais utilizadas a nível internacional destacam-se as variáveis sexo e idade apesar de raramente serem utilizadas de forma isolada [14-17]. Li et al. [18] e Kim e Kim [19] acrescentam, às variáveis sexo e idade, o estado de incapacidade como determinante da utilização de cuidados continuados. Borrayo et al. [20] sustentam que a utilização de cuidados continuados é também influenciada pelo estado cognitivo, funcional, mental e físico. De um modo geral, considera-se que utentes com maiores dificuldades em realizar actividades de vida diária, menores níveis de autonomia física e funcional e piores estados cognitivos e mentais têm maior probabilidade de utilização de cuidados continuado [21], apresentando uma maior probabilidade de internamento, justificada pela necessidade de maior intervenção para recuperação de autonomia [22].

A taxonomia referida em estudos anteriores para identificação de fatores associados ao consumo de recursos segue a abordagem definida nos trabalhos realizados por Andersen e Newman [23] e Andersen [24], que consideram três componentes principais: fatores predisponentes, condições propícias e fatores associados a necessidades específicas [20,25-27]. Na categoria dos fatores predisponentes estão incluídas as características individuais tal como a etnia, a idade e o estado civil [25]. O rendimento e a educação são variáveis incluídas na categoria referente a condições propícias [26]. E na categoria de necessidades específicas incluem-se os fatores relacionados com doenças e incapacidades, bem como limitações na reali- zação de atividades de vida diária, estado cognitivo, mental, funcional e físico [20].

Na RNCCI, parte desta informação é recolhida através do Instrumento de Avaliação Integral (IAI), composto por um conjunto de escalas de avaliação aplicada aos utentes nas dimensões biológica, psicológica e social (biopsico-social). Este instrumento contém informação recolhida periodicamente dos utentes internados em Unidades de Convalescença (UC), Unidades de Média Duração e Reabilitação (UMDR), Unidades de Longa Duração e Manutenção (ULDM) e Unidades de Cuidados Paliativos (UCP).

De acordo com a Unidade de Missão para os Cuidados Continuados [28], o objetivo do IAI consiste em avaliar um conjunto de perturbações físicas, funcionais, mentais e sociais que influenciam a autonomia dos indivíduos e que podendo ser intervencionadas são utilizadas para a construção de um plano individual de intervenção. O IAI foi desenvolvido também como instrumento que permite monitorizar a evolução do doente de forma uniformizada e passível de registo informatizado. Foi construído com base em instrumentos internacionais validados, como por exemplo o Índice de Katz utilizado para medir a autonomia física [28]. Alguns dos atributos e instrumentos utilizados no IAI já tinham sido identificados por Botelho [29] num estudo que pretendia efetuar uma avaliação multidimensional de carácter funcional, biológico, mental e social em idosos. Especificamente o IAI incorporou parte das variáveis identificadas por Botelho [29] medindo-as através dos instrumentos utilizados nesse estudo ou por adaptações aos mesmos.

Perante o enquadramento exposto e, em concomitância com a ausência de estudos nesta área em Portugal, justifica-se a realização de um trabalho de investigação que tem como objetivos estimar o consumo de recursos, medido através da duração de internamento em unidades de internamento em cuidados continuados (UICC) em Portugal, e analisar a sua associação com as características dos utentes e dos prestadores de cuidados.

\section{Métodos}

Desenho do Estudo

Foi desenvolvido um estudo transversal e retrospetivo para estimar o consumo de recursos em unidades de internamento em cuidados continuados (UICC), medido através da duração de internamento. A relação entre o consumo de recursos e as características dos utentes foi aferida através de regressão linear múltipla, tendo-se especificado dois tipos de modelos: incluindo apenas as características biopsicossociais dos utentes ou incluindo também as características dos prestadores. 
Fontes de Informação e Critérios de Exclusão

Os dados utilizados no estudo foram extraídos do sistema de informação "GestCare Cuidados Continuados Integrado" desenvolvido pela RNCCI, tendo sido cedidos pela Administração Central do Sistema de Saúde (ACSS) de forma anonimizada.

Consideraram-se todos os episódios de internamento das Unidades de Convalescença (UC), Unidades de Média Duração e Reabilitação (UMDR) e Unidades de Longa Duração e Manutenção (ULDM) em que se verificasse cumulativamente a admissão e alta nos anos 2010-2012.

Para cada episódio obteve-se informação relativa a: sexo, idade, queixas de saúde, risco de quedas, locomoção, autonomia física, estado cognitivo, tipologia de unidade, região de saúde e número de dias de internamento. Sistematiza-se na Tabela 1 as variáveis consideradas no nosso estudo que correspondem aos scores dos domínios do IAI. A forma de obtenção destes scores resulta da combinação das variáveis específicas que compõem cada um dos domínios. A informação que nos foi disponibilizada apenas continha os scores dos domínios, previamente calculados pelas regras que se explicitam na Tabela 1 .

Durante a fase de validação da consistência da informação foram excluídos: (1) 212 episódios cujo tempo de internamento era inferior a 0 dias; (2) 13.505 episódios cujo número de identificação se repetia apesar de se tratar de episódios em unidades distintas; (3) 1.883 episódios em que a data de sinalização do doente à RNCCI era posterior à data de admissão ao internamento. A amostra final foi constituída por 30.090 episódios, relativos a 28.164 utentes.

\section{Análise Descritiva e Modelos de Regressão}

A análise descritiva das variáveis nominais foi realizada através da sua distribuição, frequência absoluta e relativa.

Para a duração de internamento, foram utilizadas medidas de tendência central e medidas de dispersão tendo-se recorrido a análise de variância a dois fatores (ANOVA two-way) e ao teste não paramétrico de Kruskal-Wallis para estudar a associação entre as variáveis região e tipologia de unidade.

Uma vez que a distribuição da duração de internamento por região poderia ser influenciada pela expressão relativa de cada tipologia em cada região, prosseguiu-se a análise descritiva com o estudo da variância a dois fatores (região e tipologia) para a variável dependente transformada pelo seu logaritmo natural.

A transformação da variável dependente pelo seu logaritmo natural permitiu melhorar as propriedades estatísticas da variável natural e interpretar os coeficientes de regressão como variações percentuais, uma vez que se constatou que o tempo de internamento apresentava uma distribuição assimétrica à esquerda e leptocúrtica e a homogeneidade das variâncias não podia ser assumida pelo teste de Levene, tendo-se desta forma optado pela utilização do logaritmo natural para transformação da variável.

A existência de diferenças significativas entre a duração média de internamento por região e por tipologia e que para uma probabilidade de erro tipo I de 5\%, evidenciou uma interação significativa entre o fator tipologia e o fator região, i.e., o fator tipologia influenciava a resposta da variável dependente ao fator região.

A utilização do teste não paramétrico de Kruskal-Wallis permitiu concluir, para todos os pares possíveis de variável dependente/ variável independente e para qualquer nível de significância
( $p<0,001)$, que existiam diferenças na duração média de internamento entre as classes que compõem cada uma das variáveis independentes.

No sentido de avaliar a relação funcional entre a duração de internamento e os seus preditores, desenvolveram-se dois modelos de regressão linear múltipla.

O modelo 1, onde se avalia o impacto das características individuais dos utentes na variabilidade do tempo de internamento não considerando ou controlando as variáveis associadas às características dos prestadores de cuidados.

O modelo 2 introduz também as características da estrutura assistencial, designadamente tipologia e região de saúde em que as unidades de internamento estão implementadas, permitindo aferir o impacto das características individuais dos utentes na variabilidade do tempo de internamento, controlando as diferenças existentes entre tipologias e regiões.

Faz-se notar que o estado funcional dos utentes, medido através da autonomia física no momento da primeira avaliação, apesar de apresentado na estatística descritiva, não foi incluído nos modelos adotados. Tal deveu-se ao facto de para as duas classes mais prevalentes (incapaz e dependente), se ter detetado valores dos fatores de inflação da variância bastante superiores aos limiares máximos comummente aceites (VIF $\leq 10)$ [32], indiciando a presença de multi-colineariedade.

Para analisar a importância das características individuais na determinação do consumo de recursos, a especificação dos modelos anteriormente referidos segue uma regressão linear múltipla realizada pelo método dos mínimos quadrados ordinários do tipo $\mathrm{Yj}=\beta 0+\beta \mathrm{i} . \mathrm{Xj}+\varepsilon \mathrm{j}(\mathrm{j}=1, \ldots, n)$ em que: $\mathrm{Yj}=$ logaritmo natural da duração de internamento; $\beta 0=$ constante; $\beta \mathrm{i}=$ medida da influência de Xi em Y; Xij = vetor das variáveis independentes; $\varepsilon j=$ erro.

Todas as variáveis independentes Xij são categóricas, tendo-se optado por dicotomizar cada uma das classes de cada variável independente, assumido como classes de referência a tipologia convalescença, a região de saúde Norte, o sexo masculino, o grupo etário 18-49 anos, os episódios cujos utentes na primeira avaliação biopsicossocial não apresentaram queixas de saúde, o risco de quedas bom, a capacidade de locomoção independente e o estado cognitivo bom.

Assim, por exemplo para a variável sexo, o expoente do coeficiente $\beta$ i subtraído de 1 e multiplicado por 100, deve ser interpretado como a alteração percentual em Yj (dias de internamento) associada ao sexo feminino, i.e., a percentagem de dias de internamento que os utentes do sexo feminino têm a mais (ou a menos) do que o sexo masculino, mantendo tudo o resto constante.

Para lidar com a falta de normalidade e homocedasticidade dos resíduos foram considerados os erros padrão robustos estimados pelo método de Huber-White. Foi considerada uma probabilidade de erro de tipo I de 0,05.

Por último e como forma de especificar a análise desenvolvida, optou-se por desenvolver adicionalmente três modelos de regressão, um por cada tipologia de unidade, em que se consideraram todas as características individuais dos utentes controlando as diferenças regionais existentes.

A análise de dados foi efetuada recorrendo aos softwares estatísticos SPSS V20.0 e Stata v13.0. 
Table 1. Descrição das variáveis em estudo

\begin{tabular}{|c|c|c|}
\hline Composição da variável & Estratificação & Score/Métrica \\
\hline $\begin{array}{l}\text { Estratificação em } 2 \text { classes baseada numa variável } \\
\text { "sexo do utente" }\end{array}$ & $\begin{array}{l}0-\text { Feminino } \\
1-\text { Masculino }\end{array}$ & $\begin{array}{l}\text { Valor observado na variável } \\
\text { sexo }\end{array}$ \\
\hline $\begin{array}{l}\text { Estratificação em } 4 \text { classes baseada na faixa etária } \\
\text { do utente }\end{array}$ & $\begin{array}{l}0-80 \text { anos ou mais } \\
1-65-79 \text { anos } \\
2-50-64 \text { anos } \\
3-18-49 \text { anos }\end{array}$ & $\begin{array}{l}\text { Valor observado na variável } \\
\text { idade }\end{array}$ \\
\hline $\begin{array}{l}\text { Queixas de saúde codificadas pelo ICPC2, } \\
\text { baseadas em } 5 \text { variáveis específicas }{ }^{\mathrm{a}}\end{array}$ & $\begin{array}{l}0 \text { - Com queixas } \\
3 \text { - Sem queixas }\end{array}$ & $\begin{array}{l}\text { Menor pontuação das } \\
\text { variáveis específicas }\end{array}$ \\
\hline $\begin{array}{l}\text { Ocorrência de quedas anteriores, circunstâncias } \\
\text { em que ocorreram e sequelas associadas, baseadas } \\
\text { em } 4 \text { variáveis específicas }{ }^{b}\end{array}$ & $\begin{array}{l}0 \text { - Mau } \\
1 \text { - Insatisfatório } \\
2 \text { - Satisfatório } \\
3 \text { - Bom }\end{array}$ & $\begin{array}{l}\text { Média da pontuação das } \\
\text { variáveis específicas }\end{array}$ \\
\hline $\begin{array}{l}\text { Capacidade de locomoção do indivíduo e } \\
\text { dependência face a meios, baseadas em } 4 \text { variáveis } \\
\text { específicas }^{c} \\
\text { Adaptado de Botelho [29] }\end{array}$ & $\begin{array}{l}0 \text { - Incapaz } \\
1 \text { - Dependente } \\
2 \text { - Independente } \\
3 \text { - Autónomo }\end{array}$ & $\begin{array}{l}\text { Menor pontuação das } \\
\text { variáveis específicas }\end{array}$ \\
\hline 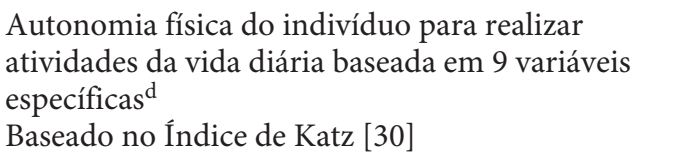 & $\begin{array}{l}0 \text { - Incapaz } \\
1 \text { - Dependente } \\
2 \text { - Independente } \\
3 \text { - Autónomo }\end{array}$ & $\begin{array}{l}\text { Menor pontuação das } \\
\text { variáveis específicas }\end{array}$ \\
\hline $\begin{array}{l}\text { Orientação do indivíduo no espaço e no tempo } \\
\text { Excerto do Mini Mental State proposto por } \\
\text { Folstein et al. [31] }\end{array}$ & $\begin{array}{l}0 \text { - Mau } \\
1 \text { - Insatisfatório } \\
2 \text { - Satisfatório } \\
3 \text { - Bom }\end{array}$ & $\begin{array}{l}\text { Média da pontuação das } \\
\text { variáveis específicas }\end{array}$ \\
\hline Tipologia de unidade na RNCCI & $\begin{array}{l}\text { Unidade de longa duração } \\
\text { Unidade de média duração } \\
\text { Unidade de convalescença }\end{array}$ & $\begin{array}{l}\text { Valor observado na variável } \\
\text { tipo de unidade }\end{array}$ \\
\hline $\begin{array}{l}\text { Identifica qual a região de saúde onde ocorreu } \\
\text { cada episódio segundo a localização da unidade } \\
\text { prestadora }\end{array}$ & $\begin{array}{l}\text { Norte } \\
\text { Centro } \\
\text { LVT } \\
\text { Alentejo } \\
\text { Algarve }\end{array}$ & $\begin{array}{l}\text { Valor observado na variável } \\
\text { região de saúde }\end{array}$ \\
\hline $\begin{array}{l}\text { Número de dias de permanência em lugar da } \\
\text { rede em cada episódio, divididos em } 3 \text { níveis } \\
\text { que correspondem à utilização prevista para cada } \\
\text { tipo de unidade }\end{array}$ & Dias de internamento & $\begin{array}{l}\text { Número de dias de } \\
\text { internamento }\end{array}$ \\
\hline
\end{tabular}

${ }^{\text {a }}$ Queixas músculo - esqueléticas, de visão, de audição, de pele e de outros órgãos/sistemas. ${ }^{b}$ Número de quedas no último ano, momento de queda, motivo de queda e sequelas de queda. ${ }^{c}$ Andar em casa, andar na rua, utilizar escadas e locomoção e meios para visão e audição. ${ }^{d}$ Lavar-se/tomar banho, vestir-se/despir-se, usar a sanita e/ou bacio/urinol, deitar-se/levantar-se da cama, sentar-se/levantar-se de cadeiras, controlar a urina, controlar as fezes, alimentar-se/comer e autonomia física e meios para visão e audição.

\section{Apresentação de Resultados}

A amostra considerada no presente estudo foi de 30.090 episódios de internamento em unidades de internamento de CCI associados a 28.164 utentes.
No respeitante às características da amostra (Tabela 2), verifica-se uma maior prevalência de episódios associados à tipologia de convalescença e às regiões Norte e Centro do país. 
Table 2. Características da amostra

\begin{tabular}{|c|c|c|c|c|c|}
\hline Variável & $n$ & $\%$ & & $n$ & $\%$ \\
\hline Sexo & & & Queixas de saúde & & \\
\hline Feminino & 17.292 & 57,5 & Com queixas & 17.036 & 56,6 \\
\hline Masculino & 12.798 & 42,5 & Sem queixas & 12.824 & 42,6 \\
\hline Grupo etário & & & Missing & 230 & 0,8 \\
\hline $18-50$ anos & 1.558 & 5,2 & Risco de quedas & & \\
\hline $50-65$ anos & 4.220 & 14,0 & Bom & 19.510 & 64,8 \\
\hline $65-80$ anos & 12.416 & 41,3 & Satisfatório & 179 & 0,6 \\
\hline$\geq 80$ anos & 11.896 & 39,5 & Insatisfatório & 4.498 & 14,9 \\
\hline Tipologia & & & Mau & 5.903 & 19,6 \\
\hline Convalescença & 13.948 & 46,4 & Locomoção & & \\
\hline Média duração & 9.959 & 33,1 & Independente & 1.614 & 5,4 \\
\hline Longa duração & 6.183 & 20,5 & Autónomo & 2.542 & 8,4 \\
\hline Duração de internamento & & & Dependente & 7.175 & 23,8 \\
\hline$\leq 30$ dias & 13.177 & 43,8 & Incapaz & 18.759 & 62,3 \\
\hline 30-90 dias & 12.054 & 40,1 & Autonomia física & & \\
\hline$>90$ dias & 4.859 & 16,1 & Independente & 220 & 0,7 \\
\hline Região & & & Autónomo & 685 & 2,3 \\
\hline Alentejo & 2.943 & 9,8 & Dependente & 16.175 & 53,8 \\
\hline Algarve & 358 & 1,2 & Incapaz & 13.010 & 43,2 \\
\hline Centro & 9.000 & 29,9 & Estado cognitivo & & \\
\hline Lisboa e V. Tejo & 4.221 & 14,0 & Bom & 11.912 & 39,6 \\
\hline \multirow[t]{3}{*}{ Norte } & 13.568 & 45,1 & Satisfatório & 3.959 & 13,2 \\
\hline & & & Insatisfatório & 3.804 & 12,6 \\
\hline & & & Mau & 10.415 & 34,6 \\
\hline
\end{tabular}

Table 3. Demora-média de internamento por tipologia e região

\begin{tabular}{lrrc}
\hline & Média & $\begin{array}{r}\text { Desvio } \\
\text { padrão }\end{array}$ & $\begin{array}{l}\text { Coeficiente de } \\
\text { variação, \% }\end{array}$ \\
\hline Total & 65,47 & 73,85 & 112,8 \\
\hline Tipologia & & & \\
$\quad$ Convalescença & 34,22 & 22,76 & 66,5 \\
$\quad$ Média duração & 84,09 & 53,17 & 63,2 \\
Longa duração & 106,01 & 128,13 & 120,9 \\
\hline Região & & & \\
Alentejo & 67,62 & 60,87 & 90,0 \\
Algarve & 57,07 & 81,61 & 143,0 \\
Centro & 75,16 & 74,50 & 99,1 \\
Lisboa e V. Tejo & 80,07 & 87,73 & 109,6 \\
$\quad$ Norte & 54,26 & 69,17 & 127,5 \\
\hline
\end{tabular}

Observou-se também uma percentagem mais elevada de episódios associados a utentes do sexo feminino, com idade igual ou superior a 65 anos, que apresentam queixas de saúde, com uma avaliação boa relativamente ao risco de quedas, maioritariamente incapazes no que respeita à sua capacidade de locomoção, dependentes ou incapazes no que respeita à sua autonomia física e com uma concentração elevada nas classes extremas no que respeita ao estado cognitivo.

Através da Tabela 3, podemos observar os resultados descritivos das variáveis em estudo. Assim, pode-se constatar que a duração de internamento apresentou uma dispersão significativa, independentemente da desagregação adotada. Em média, a duração de internamento nas unidades de convalescença foi de 34,2 dias, 84,1 dias nas unidades de média duração e reabilitação e 106 dias nas unidades de longa duração e manutenção, comportamento esperado considerando os critérios de referenciação estabelecidos. De notar que a duração média de internamento nas unidades de convalescença foi superior ao tempo de internamento máximo referencial adotado (30 dias): $28,6 \%$ dos episódios nas unidades de convalescença apresentaram tempo de internamento superior a 30 dias.

A desagregação por região de saúde também permitiu constatar a variabilidade da duração média de internamento, verificando-se que a região mais representada (Norte) foi também onde se observou uma duração média de internamento mais reduzida (54,3 dias). 
Table 4. Resultados das regressões lineares (duração de internamento e características biopsicossociais)

\begin{tabular}{|c|c|c|c|}
\hline \multicolumn{2}{|c|}{ Var. dependente: Log da duração de internamento } & \multirow{2}{*}{$\begin{array}{l}\begin{array}{l}\text { Modelo } 1 \\
\text { (sem tipologia e região } \\
\text { do prestador) }\end{array} \\
\begin{array}{l}0,0728^{* * *} \\
{[0,0109]}\end{array}\end{array}$} & \multirow{2}{*}{$\begin{array}{l}\text { Modelo } 2 \\
\text { (incluindo tipologia e } \\
\text { região do prestador) }\end{array}$} \\
\hline $\begin{array}{l}\text { Sexo } \\
\text { (ref. Masculino) }\end{array}$ & Feminino & & \\
\hline $\begin{array}{l}\text { Grupo etário } \\
\text { (ref. 18-49 anos) }\end{array}$ & $\begin{array}{l}50-64 \text { anos } \\
65-79 \text { anos } \\
\geq 80 \text { anos }\end{array}$ & $\begin{array}{l}0,0278 \\
{[0,0287]} \\
0,00335 \\
{[0,0266]} \\
-0,0113 \\
{[0,0273]}\end{array}$ & $\begin{array}{l}0,104^{* * *} \\
{[0,0263]} \\
0,0648^{* * *} \\
{[0,0246]} \\
0,00639 \\
{[0,0253]}\end{array}$ \\
\hline $\begin{array}{l}\text { Queixas de saúde } \\
\text { (ref. Sem Queixas) }\end{array}$ & Com Queixas & $\begin{array}{l}-0,0134 \\
{[0,0105]}\end{array}$ & $\begin{array}{l}-0,0118 \\
{[0,00948]}\end{array}$ \\
\hline $\begin{array}{l}\text { Avaliação de quedas } \\
\text { (ref. Bom) }\end{array}$ & $\begin{array}{l}\text { Satisfatório } \\
\text { Insatisfatório } \\
\text { Mau }\end{array}$ & $\begin{array}{l}0,0474 \\
{[0,0649]} \\
0,118^{* * *} \\
{[0,0148]} \\
0,0782^{* * *} \\
{[0,0129]}\end{array}$ & $\begin{array}{l}-0,0226 \\
{[0,0590]} \\
0,0648^{* * *} \\
{[0,0132]} \\
0,0953^{* * *} \\
{[0,0115]}\end{array}$ \\
\hline $\begin{array}{l}\text { Locomoção } \\
\text { (ref. Independente) }\end{array}$ & $\begin{array}{l}\text { Autónomo } \\
\text { Dependente } \\
\text { Incapaz }\end{array}$ & $\begin{array}{l}-0,0539^{* *} \\
{[0,0259]} \\
-0,0481^{* *} \\
{[0,0233]} \\
0,0278 \\
{[0,0224]}\end{array}$ & $\begin{array}{l}-0,0220 \\
{[0,0230]} \\
-0,0318 \\
{[0,0210]} \\
-0,0325 \\
{[0,0204]}\end{array}$ \\
\hline $\begin{array}{l}\text { Cognitivo } \\
\text { (ref. Bom) }\end{array}$ & $\begin{array}{l}\text { Satisfatório } \\
\text { Insatisfatório } \\
\text { Mau }\end{array}$ & $\begin{array}{l}0,0908^{* * *} \\
{[0,0147]} \\
0,176^{* * *} \\
{[0,0163]} \\
0,208^{* * *} \\
{[0,0132]}\end{array}$ & $\begin{array}{l}-0,00215 \\
{[0,0127]} \\
-0,0214 \\
{[0,0146]} \\
-0,0980^{* * *} \\
{[0,0128]}\end{array}$ \\
\hline Constante & & $\begin{array}{l}3,615^{* * *} \\
{[0,0331]}\end{array}$ & $\begin{array}{l}3,207^{* * *} \\
{[0,0307]}\end{array}$ \\
\hline Tipologia e região & & $\mathrm{N}$ & S \\
\hline $\begin{array}{l}\text { Observações } \\
R^{2}\end{array}$ & & $\begin{array}{l}29.860 \\
0,017\end{array}$ & $\begin{array}{l}29.860 \\
0,194\end{array}$ \\
\hline
\end{tabular}

Sig. estatística: * $10 \% ; * * 5 \%$; ** $1 \%$. Erros padrão robustos.

Os resultados dos modelos de regressão utilizados para estimar a relação entre consumo de recursos e características biopsicossociais encontram-se na Tabela 4.

De uma forma geral, verifica-se que a capacidade explicativa dos modelos é relativamente reduzida, uma vez que os seus coeficientes de determinação são de 0,017 para o modelo 1 e que, embora a inclusão das características relativas à oferta aumente significativamente a capa- cidade do modelo, esta ainda se mantém relativamente reduzida $(0,194)$.

Da análise da relação entre variáveis, verifica-se que no modelo 1, o sexo feminino apresenta um tempo de internamento $7,6 \%$ superior ao verificado no sexo masculino $(p<0,01)$ mantendo todas as restantes características individuais constantes. O controlo das diferenças da duração de internamento associadas à oferta assistencial (mo- 
Table 5. Resultados das regressões lineares por tipologia de unidade (duração de internamento e características biopsicossociais)

\begin{tabular}{|c|c|c|c|c|}
\hline \multicolumn{2}{|c|}{$\begin{array}{l}\text { Var. dependente: Log da duração de } \\
\text { internamento }\end{array}$} & \multirow{2}{*}{$\begin{array}{l}\text { Convalescença } \\
0,0543^{* * *} \\
{[0,00974]}\end{array}$} & \multirow{2}{*}{$\begin{array}{l}\text { Média duração } \\
0,140^{* * *} \\
{[0,0178]}\end{array}$} & \multirow{2}{*}{$\begin{array}{l}\text { Longa duração } \\
0,136^{* * *} \\
{[0,0317]}\end{array}$} \\
\hline $\begin{array}{l}\text { Sexo } \\
\text { (ref. Masculino) }\end{array}$ & Feminino & & & \\
\hline $\begin{array}{l}\text { Grupo etário } \\
\text { (ref. 18-49 anos) }\end{array}$ & $\begin{array}{l}50-64 \text { anos } \\
65-79 \text { anos } \\
\geq 80 \text { anos }\end{array}$ & $\begin{array}{l}0,105^{* * *} \\
{[0,0272]} \\
0,0962 * * * \\
{[0,0261]} \\
0,0417 \\
{[0,0272]}\end{array}$ & $\begin{array}{l}0,119^{* * *} \\
{[0,0444]} \\
0,0666^{*} \\
{[0,0402]} \\
-0,00861 \\
{[0,0410]}\end{array}$ & $\begin{array}{l}0,107 \\
{[0,107]} \\
0,00421 \\
{[0,0932]} \\
-0,0391 \\
{[0,0922]}\end{array}$ \\
\hline $\begin{array}{l}\text { Queixas de saúde } \\
\text { (ref. Sem queixas) }\end{array}$ & Com Queixas & $\begin{array}{l}-0,0257^{* * *} \\
{[0,00905]}\end{array}$ & $\begin{array}{l}-0,00440 \\
{[0,0175]}\end{array}$ & $\begin{array}{l}0,00309 \\
{[0,0325]}\end{array}$ \\
\hline $\begin{array}{l}\text { Avaliação de quedas } \\
\text { (ref. Bom) }\end{array}$ & $\begin{array}{l}\text { Satisfatório } \\
\text { Insatisfatório } \\
\text { Mau }\end{array}$ & $\begin{array}{l}-0,0148 \\
{[0,0525]} \\
0,0237^{*} \\
{[0,0143]} \\
0,0747^{* * *} \\
{[0,0113]}\end{array}$ & $\begin{array}{l}-0,151 \\
{[0,128]} \\
0,0930^{* * *} \\
{[0,0229]} \\
0,0967^{* * *} \\
{[0,0204]}\end{array}$ & $\begin{array}{l}0,108 \\
{[0,133]} \\
0,0943^{* *} \\
{[0,0409]} \\
0,127^{* * *} \\
{[0,0471]}\end{array}$ \\
\hline $\begin{array}{l}\text { Locomoção } \\
\text { (ref. Independente) }\end{array}$ & $\begin{array}{l}\text { Autónomo } \\
\text { Dependente } \\
\text { Incapaz }\end{array}$ & $\begin{array}{l}-0,0124 \\
{[0,0216]} \\
-0,0324 \\
{[0,0206]} \\
-0,00504 \\
{[0,0200]} \\
\end{array}$ & $\begin{array}{l}-0,0376 \\
{[0,0444]} \\
-0,0334 \\
{[0,0375]} \\
-0,0521 \\
{[0,0352]}\end{array}$ & $\begin{array}{l}0,124 \\
{[0,144]} \\
0,0914 \\
{[0,126]} \\
0,107 \\
{[0,123]} \\
\end{array}$ \\
\hline $\begin{array}{l}\text { Cognitivo } \\
\text { (ref. Bom) }\end{array}$ & $\begin{array}{l}\text { Satisfatório } \\
\text { Insatisfatório } \\
\text { Mau }\end{array}$ & $\begin{array}{l}-0,00202 \\
{[0,0129]} \\
-0,0611^{* * *} \\
{[0,0167]} \\
-0,0878^{* * *} \\
{[0,0156]}\end{array}$ & $\begin{array}{l}0,0131 \\
{[0,0241]} \\
-0,0106 \\
{[0,0246]} \\
-0,122^{* * *} \\
{[0,0209]}\end{array}$ & $\begin{array}{l}-0,0507 \\
{[0,0641]} \\
0,0254 \\
{[0,0549]} \\
-0,0145 \\
{[0,0424]}\end{array}$ \\
\hline Constante & & $\begin{array}{l}3,194^{* * *} \\
{[0,0319]}\end{array}$ & $\begin{array}{l}4,096^{* * *} \\
{[0,0502]}\end{array}$ & $\begin{array}{l}3,596^{* * *} \\
{[0,162]}\end{array}$ \\
\hline Região de Saúde & & $\mathrm{S}$ & $\mathrm{S}$ & $\mathrm{S}$ \\
\hline $\begin{array}{l}\text { Observações } \\
R^{2}\end{array}$ & & $\begin{array}{l}13.877 \\
0,082\end{array}$ & $\begin{array}{l}9.862 \\
0,023\end{array}$ & $\begin{array}{l}6.121 \\
0,038\end{array}$ \\
\hline
\end{tabular}

Sig. estatística: ${ }^{*} 10 \% ; * * 5 \% ; * * 1 \%$. Erros padrão robustos.

delo 2), permite também concluir que o sexo feminino apresenta um tempo de internamento $10,7 \%$ superior ao sexo masculino, ceteris paribus.

A variável idade apenas apresenta significância estatística para explicar a variabilidade da duração de internamento quando as características associadas à oferta são incluídas e apenas para as faixas etárias dos 50 aos 64 anos e dos 65 aos 79 anos: os utentes com idades compreendi-

Consumo de Recursos em Unidades de Cuidados Continuados das entre os 50 e os 64 anos apresentam uma duração de internamento $11 \%$ superior aos utentes com idades entre 18 e 49 anos, considerando todas as restantes características constantes.

A presença de queixas de saúde no momento da primeira avaliação biopsicossocial não apresenta significância estatística para a explicação da variabilidade da duração de internamento. 
Por sua vez, a avaliação da capacidade de locomoção sugere uma ausência de capacidade preditiva para a variabilidade da duração de internamento, apesar de, para 95\% de confiança e apenas quando as características da oferta não são controladas, existir significância estatística para as classes autónomo e dependente quando comparadas com a classe de referência independente.

Utentes avaliados com um risco de quedas insatisfatório ou mau apresentam durações de internamento significativamente superiores aos utentes sem risco de quedas.

O estado cognitivo dos utentes no momento da primeira avaliação, quando não são controladas as características da oferta de cuidados e tendo por referência os utentes com bom estado cognitivo, apresenta valores positivos e crescentes à medida que o estado cognitivo decresce, i.e., aparentemente a duração de internamento cresce com o aumento da incapacidade cognitiva. Contudo, após inclusão das variáveis referentes à tipologia e região de saúde, a relação perde relevância estatística, sendo que para o pior estado cognitivo é possível inclusive encontrar uma relação inversa, i.e., utentes com mau estado cognitivo apresentam durações de internamento 9,3\% mais reduzidas do que utentes com bom estado cognitivo, mantendo todas as restantes características constantes.

Não obstante as limitações apresentadas e considerando que a relevância das características individuais em cada tipologia poderia não ter expressão semelhante e apresentar-se como fator de confundimento, optou-se por desenvolver três modelos de regressão, um por cada tipologia, considerando todas as características individuais e controlando as diferenças regionais existentes. Os resultados destes modelos apresentam-se na Tabela 5 .

Relativamente à variável sexo, é possível confirmar que as mulheres apresentam em todas as tipologias uma duração de internamento superior aos homens, sendo que as diferenças são mais expressivas nas unidades de média duração.

A idade apenas apresenta capacidade preditiva da duração de internamento nas classes etárias 50-64 anos e 65-79 anos, sendo que para as unidades de longa duração, a idade não tem poder explicativo estatisticamente significativo em nenhuma das classes etárias.

A apresentação de queixas de saúde pelos utentes no momento da primeira avaliação biopsicossocial em unidades de convalescença apresenta-se como fator protetor relativamente à duração de internamento, i.e., estes utentes apresentam uma duração de internamento 2,5\% inferior aos utentes que não apresentam queixas.

A capacidade de locomoção não apresenta capacidade preditiva para nenhuma das tipologias de cuidados.
Utentes com avaliação de risco de quedas insatisfatório ou mau apresentam tempos de internamento significativamente superiores aos utentes cujo risco é avaliado como bom em todas as tipologias de cuidados, sendo que este fator apresenta maior capacidade discriminatória nas unidades de longa duração.

Relativamente ao estado cognitivo é possível concluir que não apresenta capacidade preditiva da duração de internamento nas unidades de longa duração. Contudo, utentes das unidades de convalescença com estado cognitivo insatisfatório ou mau e utentes das unidades de média duração com estado cognitivo mau, apresentam durações de internamento significativamente mais reduzidas do que utentes com bom estado cognitivo.

A desagregação da análise por tipologia de internamento parece confirmar que as características individuais consideradas apresentam reduzida capacidade preditiva da variabilidade da duração de internamento, o que se encontra plasmado nos coeficientes de determinação reduzidos que foram obtidos.

\section{Discussão}

Os resultados apurados sugerem que o consumo de recursos está maioritariamente associado às características dos prestadores de cuidados. De facto, a inclusão da tipologia e da região no modelo de regressão aumentou significativamente a capacidade preditiva do modelo $(0,194)$, sugerindo que as características dos prestadores de cuidados e o condicionamento da referenciação dos utentes em função da estrutura existente têm um poder explicativo substancialmente mais elevado do que as características individuais dos utentes. Num estudo semelhante, verificou-se que as características da oferta permitem explicar cerca de $24 \%$ da variação do consumo de recursos [33].

No que diz respeito às características dos utentes, verificamos que apenas a variável sexo apresenta capacidade preditiva do consumo de recursos em todos os modelos, sendo que o sexo feminino apresenta uma duração de internamento superior à do sexo masculino. Este resultado é consistente com outros estudos que, em contexto de reabilitação de acidente vascular cerebral, sustentam que estas diferenças de género podem ser explicadas pelo facto de as mulheres apresentarem piores estados funcionais na admissão, menores apoios sociais, existindo também um maior número de mulheres com estado civil de viuvez e a viver sozinhas [34]. 
A avaliação do risco de quedas apresentou capacidade preditiva da duração de internamento. Os utentes com risco de queda insatisfatório ou mau encontram-se associados a episódios com maiores durações de internamento. Esta associação apresenta significância estatística em todas as tipologias, sendo que os utentes com maior risco de quedas internados em unidades de longa duração apresentavam mais $13,5 \%$ dias de internamento do que os utentes avaliados com risco de queda classificado como bom. Importa realçar que os resultados obtidos são independentes da ocorrência efetiva de queda durante o episódio de internamento. No entanto, um historial de quedas e fraturas é um dos maiores preditores de ocorrência futura de quedas [35]. Diversos estudos demonstram que existe uma relação entre a ocorrência de quedas e a duração de internamento, verificando-se custos e durações de internamentos mais elevados nos utentes com quedas [36-38]. Existe ainda a possibilidade destes utentes beneficiarem de medidas protetoras que prolongam o tempo de reabilitação e condicionam a possibilidade de uma alta mais precoce para o domicílio.

A idade apresentou igualmente uma associação positiva com a duração de internamento, isto é, escalões etários mais elevados apresentam maior duração de internamento. Contudo, esta associação só é estatisticamente significativa quando as características dos prestadores de cuidados são controladas. A desagregação da análise por tipologia permitiu ainda concluir que as diferenças só são estatisticamente significativas para os escalões etários dos 50 aos 64 anos e dos 64 aos 79 anos e apenas para as tipologias convalescença e média duração. Assim, para as unidades de longa duração a idade parece não influenciar a duração de internamento. Um estudo realizado em utentes internados em cuidados continuados na Noruega, concluiu que não existe relação entre a idade e os recursos consumidos [33]. Ressalva-se que não estava disponível a idade enquanto variável contínua e que existia uma elevada concentração de episódios (mais de $80 \%$ ) nos escalões etários dos 65 a 79 anos e mais de 80 anos. Uma diferente composição dos escalões etários da amostra poderia proporcionar diferentes resultados.

O estado cognitivo apresenta uma associação inversa com a duração de internamento, desde que controladas as características dos prestadores de cuidados. Verificouse que utentes com estado cognitivo mau apresentavam menor duração de internamento. A análise por tipologia veio, no entanto, demonstrar que esta associação era apenas significativa nas tipologias de convalescença e média duração. Num estudo realizado em Espanha, por Baztán et al. [39], foram encontrados resultados semelhantes

Consumo de Recursos em Unidades de Cuidados Continuados numa unidade geriátrica de média duração. Estes resultados podem ser explicados por duas potenciais razões. Baztán et al. [39], Valderrama-Gama et al. [40], e Patrick et al. [41] concluem que os utentes com pior estado cognitivo têm um menor tempo de internamento porque apresentam menor capacidade de reabilitação funcional. A possibilidade de estes utentes terem uma maior mobilidade na rede, transitando de unidades com cuidados reabilitativos para unidades com cuidados de manutenção, poderá também explicar estes resultados. Estudos posteriores devem ser efetuados para validar esta potencial associação.

O conjunto de resultados apurados e suas implicações deverão ser interpretados com alguma prudência, nomeadamente pelas limitações impostas ao nível da exaustividade e fiabilidade da informação disponível. A falta de explicitação de alguns conceitos básicos (por exemplo o significado de um episódio de internamento), a variabilidade das práticas de registo ou a utilização do IAI como instrumento de registo das características dos utentes poderão constituir fatores de relevo neste contexto. No entanto, os autores acreditam que foi utilizada a melhor informação disponível para a prossecução dos objectivos do estudo.

A este respeito importa salientar que em países como os EUA, onde foram desenvolvidos processos sistemáticos de recolha dados no âmbito do Long-Term Care, recorre-se de uma forma generalizada ao Minimum Data Set desde 1987 [13]. Noutros países é utilizado o interRAI LTCF [42]. Para além de serem instrumentos geralmente aceites neste contexto, são uniformizados, tendo sido testados e validados em diversos contextos e realidades. A sua aplicação permitiria ainda efetuar comparações e sobretudo promover o uso do sistema de classificação de utentes mais comummente referido internacionalmente para este fim: os Resource Utilization Groups (RUGs). Os RUGs são um sistema de classificação de utentes, desenvolvido nos EUA na década de 80 do século passado, que pretende agrupar os utentes em categorias homogéneas de utilização diária de recursos $[43,44]$.

O recurso a um sistema de classificação de utentes na área dos cuidados continuados no nosso país parece ser crucial para um melhor entendimento sobre a distribuição do consumo de recursos nas unidades prestadoras. Recorde-se que desta aplicação dependem outros desenvolvimentos fundamentais para garantir a utilização de instrumentos de gestão que promovam a criação de valor, entre os quais se destacam a estratificação populacional, a análise da produção, o financiamento ou a avaliação de desempenho. 


\section{Conclusão}

Os crescentes desafios colocados à prestação de cuidados a indivíduos idosos, com multipatologia crónica e com dependência conduziram a uma resposta organizacional que em Portugal foi consubstanciada na criação da RNCCI. Contudo, os instrumentos de apoio, bem como estudos que incidam sobre a distribuição do consumo de recursos nas unidades que constituem a RNCCI são praticamente inexistentes.

Desta forma, este estudo estimou o consumo de recursos, medido através da duração de internamento, em unidades de internamento nos cuidados continuados em Portugal e analisou a sua associação às características dos prestadores e dos utentes.

$\mathrm{Na}$ amostra e período considerados verificou-se que o tempo médio de internamento foi de 34,2 dias nas unidades de convalescença, 84,1 dias nas unidades de média duração e reabilitação e 106 dias nas unidades de longa duração e manutenção. Verificámos a existência de assimetrias regionais relativamente ao tempo médio de inter- namento refletindo as diferenças de resposta assistencial disponível em cada região. Concluímos também pela existência de uma dispersão significativa independentemente da tipologia ou região considerada.

Após a análise dos modelos de regressão linear múltipla, os resultados apurados sugerem que o tempo de internamento é influenciado sobretudo pelas características dos prestadores de cuidados e não pelas características dos utentes.

A resposta a esta realidade exige um acompanhamento adequado e a consideração de ferramentas e instrumentos de gestão, como a aplicação de um novo sistema de classificação de utentes. A aplicação de um sistema de classificação poderia, para além de garantir uma classificação homogénea das características dos utentes, contribuir para um melhor entendimento sobre a distribuição do consumo de recursos e ter impactos significativos noutras áreas relevantes como a estratificação do risco, sistema de financiamento, garantia da qualidade e avaliação de desempenho.

\section{References}

1 Schulz E: The Long-Term Care System for the Elderly in Germany. Brussels, European Network of Economic Policy Research Institutes (ENEPRI), 2010.

2 McGregor M, Ronald L: Residential longterm care for Canadian seniors: nonprofit, for-profit or does it matter? Montreal, Institute for Research on Public Policy, 2011, Report No.: 978-0-88645-237-7.

3 Lezovic M, Raucinova M, Kovac A, Moricova $\mathrm{S}$, Kovac R: Long-term care in developed countries and recommendations for Slovak Republic. Cent Eur J Public Health 2008;16: 21-25.

4 Gutiérrez F: The Spanish Long-Term Care System. Brussels, European Network of Economic Policy Research Institutes (ENEPRI), 2010.

5 Comas-Herrera A: The Long-Term Care System for the Elderly in England. Brussels, European Network of Economic Policy Research Institutes (ENEPRI), 2010.

6 Brown J, Finkelstein A: Insuring long-term care in the United States. J Econ Perspect 2011;25:119-142.

7 Fernandez JL, Forder J, Knapp M: Long term care; in Glied S, Smith P (eds): The Oxford Handbook of Health Economics. Oxford, Oxford University Press, 2011, pp 578-601.

8 Decreto-Lei no 101/2006. DR. Série I - A 109 (2006.06.06) 3856-3865. Procede à criação da Rede Nacional de Cuidados Continuados Integrados.
9 OECD: Long-term Care for Older People. Paris, OECD Publishing, 2005, DOI: 10.1787/ 9789264015852-en.

10 Portugal, Ministério da Saúde, ACSS: Monitorização da Rede Nacional de Cuidados Continuados Integrados (RNCCI). Lisboa, Administração Central do Sistema de Saúde (ACSS), 2015.

11 Federal Ministry of Health: Selected Facts and Figures about Long Term Care Insurance (online). Berlin, Federal Ministry of Health, 2012. Available from: http://www.bmg.bund. de/fileadmin/dateien/Englische_Dateien/ 121116_Factsheet_Long-Term_Care_Insurance.pdf (accessed April 1, 2016).

12 OECD: Public long-term care financing arrangements in OECD countries: help wanted?: Providing and paying for long-term care (online). Paris, OECD, 2011. Available from: http://www.oecd.org/health/health-systems/ helpwantedprovidingandpayingforlongtermcare.html (accessed January 14, 2016).

13 Centers for Medicare and Medicaid Services: Long term care Minimum Data Set (MDS) (online). Baltimore, Centers for Medicare and Medicaid Services, 2012. Available from: https://www.cms.gov/Research-StatisticsData-and-Systems/Files-for-Order/IdentifiableDataFiles/LongTermCareMinimumDataSetMDS.html (accessed April 4, 2016).
14 Rosen AK, Reid R, Broemeling A-M, Rakovski CC: Applying a risk-adjustment framework to primary care: can we improve on existing measures? Ann Fam Med 2003;1:44-51.

15 van Barneveld EM, Lamers LM, van Vliet RC, van de Ven WP: Risk sharing as a supplement to imperfect capitation: a tradeoff between selection and efficiency. J Health Econ 2001;20: 147-168.

16 Green CA, Pope CR: Gender, psychosocial factors and the use of medical services: a longitudinal analysis. Soc Sci Med 1999;48:13631372.

17 Ash AS, Ellis RP, Pope GC, Ayanian JZ, Bates DW, Burstin $\mathrm{H}$, et al: Using diagnoses to describe populations and predict costs. Health Care Financ Rev 2000;21:7-28.

18 Li IC, Fann SL, Kuo HAT: Predictors of the utilization of long-term care (LTC) services among residents in community-based LTC facilities in Taiwan. Gerontol Geriatr 2011;53: 303-308.

19 Kim EY, Kim CY: Who wants to enter a longterm care facility in a rapidly aging non-Western society? Attitudes of older Koreans toward long-term care facilities. J Am Geriatr Soc 2004;52:2114-2119.

20 Borrayo EA, Salmon JR, Polivka L, Dunlop $\mathrm{BD}$ : Utilization across the continuum of longterm care services. Gerontologist 2002;42: 603-612. 
21 Bakx P: Determinants of long-term care use. Rotterdam, Erasmus University Rotterdam; 2010; Master Thesis in Health Economics, Policy, and Law.

22 Feigenson JS, McDowell FH, Meese P, McCarthy ML, Greenberg SD: Factors influencing outcome and length of stay in a stroke rehabilitation unit: Part 1: Analysis of 248 unscreened patients-medical and functional prognostic indicators. Stroke 1977;8:651656.

23 Andersen R, Newman JF: Societal and individual determinants of medical care utilization in the United States. Milbank Mem Fund Q Health Soc 1973;51:95-124.

24 Andersen R: Revisiting the behavioral model and access to medical care: does it matter? J Health Soc Behav 1995;36:1-10.

25 Bookwala J, Zdaniuk B, Burton L, Lind B, Jackson S, Schulz R: Concurrent and longterm predictors of older adults' use of community-based long-term care services: The Caregiver Health Effects Study. J Aging Health 2004;16:88-115.

26 Skarupski KA, McCann JJ, Bienias JL, Wolinsky FD, Aggarwal NT, Evans DA: Use of home-based formal services by adult day care clients with Alzheimer's disease. Home Health Care Serv Q 2008;27:217-239.

27 Heider D, Matschinger H, Müller H, Saum K-U, Quinzler R, Haefeli WE, et al: Health care costs in the elderly in Germany: an analysis applying Andersen's behavioral model of health care utilization. BMC Health Serv Res 2014;14:1-12.
28 Portugal, Unidade de Missão para Cuidados Continuados Integrados: Critérios de monitorização biopsicossocial na RNCCI: avaliação biopsicossocial através do Instrumento de Avaliação Integral (IAI). Lisboa, Unidade de Missão para os Cuidados Continuados Integrados, 2007. Available from: http://www. acss.minsaude.pt/Portals/0/ApresentacaoIAI_UMCCI.pdf (accessed April 27, 2016).

29 Botelho M: Autonomia funcional em idosos: caracterização multidimensional em idosos utentes de um centro de saúde urbano. Lisboa, Faculdade de Ciências Médicas. Universidade Nova de Lisboa, 1999; PhD Thesis.

30 Katz S, Ford AB, Moskowitz RW, Jackson BA, Jaffe MW: Studies of illness in the aged: the index of ADL: a standardized measure of biological and psychosocial function. JAMA 1963; 185:914-919.

31 Folstein MF, Folstein SE, McHugh PR: "Minimental state". A practical method for grading the cognitive state of patients for the clinician. J Psychiatr Res 1975;12:189-198.

32 O'brien RM: A caution regarding rules of thumb for variance inflation factors. Qual Quant 2007;41:673-690.

33 Døhl Ø, Garåsen H, Kalseth J, Magnussen J: Variations in levels of care between nursing home patients in a public health care system. BMC Health Serv Res 2014;14:1-10.

34 Kapral MK, Fang J, Hill MD, Silver F, Richards J, Jaigobin C, et al: Sex differences in stroke care and outcomes: results from the Registry of the Canadian Stroke Network. Stroke 2005;36:809-814.

35 Tinetti ME, Speechley M, Ginter SF: Risk factors for falls among elderly persons living in the community. N Engl J Med 1988;319: 1701-1707.

36 Basic D, Hartwell TJ: Falls in hospital and new placement in a nursing home among older people hospitalized with acute illness. Clin Interv Aging 2015;10:1637-1643.
37 Bouldin ED, Andresen EM, Dunton NE, Simon M, Waters TM, Liu M, et al: Falls among adult patients hospitalized in the United States: prevalence and trends. J Patient Saf 2013;9:13-17.

38 Bates DW, Pruess K, Souney P, Platt R: Serious falls in hospitalized patients: correlates and resource utilization. Am J Med 1995;99: 137-143.

39 Baztán JJ, Domenech JR, González M, Forcano S, Morales C, Ruipérez I: Ganancia funcional y estancia hospitalaria en la unidad geriátrica de media estancia del Hospital Central de Cruz Roja de Madrid. Rev Esp Salud Publica 2004;78:355-366.

40 Valderrama-Gama E, Damian J, Guallar E, Rodriguez-Manas L: Previous disability as a predictor of outcome in a geriatric rehabilitation unit. J Gerontol A Biol Sci Med Sci 1998; 53:M405-M409.

41 Patrick L, Knoefel F, Gaskowski P, Rexroth D: Medical comorbidity and rehabilitation efficiency in geriatric inpatients. J Am Geriatr Soc 2001;49:1471-1477.

42 InterRAI: Long-Term Care Facilities (LTCF) (online). Ann Arbor, Institute of Gerontology, University of Michigan. Available from: http://interrai.org/long-term-care-facilities. html (accessed January 14, 2016, updated 2016).

43 Clauser SB, Fries BE: Nursing home resident assessment and case-mix classification: Cross-national perspectives. Health Care Financ Rev 1992;13:135-155.

44 Martin L, Fries BE, Hirdes JP, James M: Using the RUG-III classification system for understanding the resource intensity of persons with intellectual disability residing in nursing homes. J Intellect Disabil 2011;15:131-141. 